Model-Based Detection of Radioactive Contraband for Harbor Defense Incorporating Compton Scattering Physics

J. V. Candy, D. H. Chambers, E. F. Breitfeller, B. L. Guidry, J. M. Verbeke, M. A. Axelrod, K. E. Sale, A. M. Meyer

March 4, 2010

IEEE OCEANS ' 10

Sydney, Australia

May 24, 2010 through May 27, 2010 
This document was prepared as an account of work sponsored by an agency of the United States government. Neither the United States government nor Lawrence Livermore National Security, LLC, nor any of their employees makes any warranty, expressed or implied, or assumes any legal liability or responsibility for the accuracy, completeness, or usefulness of any information, apparatus, product, or process disclosed, or represents that its use would not infringe privately owned rights. Reference herein to any specific commercial product, process, or service by trade name, trademark, manufacturer, or otherwise does not necessarily constitute or imply its endorsement, recommendation, or favoring by the United States government or Lawrence Livermore National Security, LLC. The views and opinions of authors expressed herein do not necessarily state or reflect those of the United States government or Lawrence Livermore National Security, LLC, and shall not be used for advertising or product endorsement purposes. 


\title{
Model-Based Detection of Radioactive Contraband for Harbor Defense Incorporating Compton Scattering Physics
}

\author{
J. V. Candy, Fellow, IEEE, D. H. Chambers, Senior Member, IEEE, E. F. Breitfeller, Member, IEEE, B. L. Guidry, \\ Member, IEEE, J. M. Verbeke, M. A. Axelrod, K. E. Sale and A. M. Meyer Senior Member, IEEE
}

\begin{abstract}
The detection of radioactive contraband is a critical problem is maintaining national security for any country. Photon emissions from threat materials challenge both detection and measurement technologies especially when concealed by various types of shielding complicating the transport physics significantly. This problem becomes especially important when ships are intercepted by U.S. Coast Guard harbor patrols searching for contraband. The development of a sequential model-based processor that captures both the underlying transport physics of gamma-ray emissions including Compton scattering and the measurement of photon energies offers a physics-based approach to attack this challenging problem. The inclusion of a basic radionuclide representation of absorbed/scattered photons at a given energy along with interarrival times is used to extract the physics information available from the noisy measurements portable radiation detection systems used to interdict contraband. It is shown that this physics representation can incorporated scattering physics leading to an "extended" model-based structure that can be used to develop an effective sequential detection technique. The resulting model-based processor is shown to perform quite well based on data obtained from a controlled experiment.
\end{abstract}

\section{INTRODUCTION}

Whether a Coast Guard patrol boat interdicting and searching a vessel in the harbor for contraband or a commercial semitrailer truck passing through a portal monitor at a reasonable speed must be screened at ports-of-entry for possible threats emanating from radioactive contraband The need to investigate techniques that can provide for more sensitive detection of terrorist threats throughout the world demand that meaningful approaches be developed to solving many critical security problems for the protection of valuable national resources.

Detection of radiological threat materials is a difficult problem primarily because of low observable count rates and short detection intervals available. For instance, semi-trailer vehicles move through portal systems allowing less than 10 seconds for the initial screening. Shielding materials from packaging and adjacent cargo present major difficulties in these low-count, hostile environments. Low-count detection is a challenging problem made difficult because of background noise, measurement system inadequacies, and the heterogeneous transport paths between source and detector [1]-[3]. Even the current methods of gamma-ray spectrometry incorporating high resolution detectors are challenged by the low-count problem.
Recall that this measurement technique estimates the energy (probability) distribution creating a count-dependent histogram at various energies (counts vs. energy) discarding arrival time information [2]. Thus, the basic problem we investigate is the detection and identification of radioactive contraband from low-count measurements using a physics-based statistical approach incorporating scattering physics based on a modelbased signal processing approach.

Some work has been accomplished on this problem, but very little effort has been performed on this specific application in the signal processing area [4]-[8]. Our approach closely follows the development of our Bayesian model-based processor of a previous paper with the incorporation of Compton scattering photons into the processing scheme [10]. This is accomplished by extending the radionuclide representation to incorporate the scattered photons along with the development of a simplified one-dimensional transport model required to capture material interactions in the shield and detector materials [9].

In this paper, we briefly develop the physics-based signal processing models employed in the subsequent model-based constructs. Here we start with the "extended" monoenergetic representation incorporating Compton scattering physics and then incorporate noise and parameter uncertainties into the model. A simplified one-dimensional transport model is discussed to capture photon scattering physics in both shield and detector materials. Based on these representations, we develop the sequential detection/identification paradigm and then investigate solutions to the processing problem and implementation of the technique. The results of applying the processor to controlled experimental data shows the capability of the sequential processor to perform in a reasonable manner.

In Sec. II we develop the physics-based signal processing models employed in the subsequent Bayesian constructs. Next we develop the sequential detection paradigm in Sec. III. In Sec. IV we investigate solutions to the processing problem and implementation of the technique. In Sec. V we develop the overall identification scheme and demonstrate its performance on experimental data. The results of applying the processor to controlled experimental data shows the capability of the sequential processor to perform in an effective manner. 

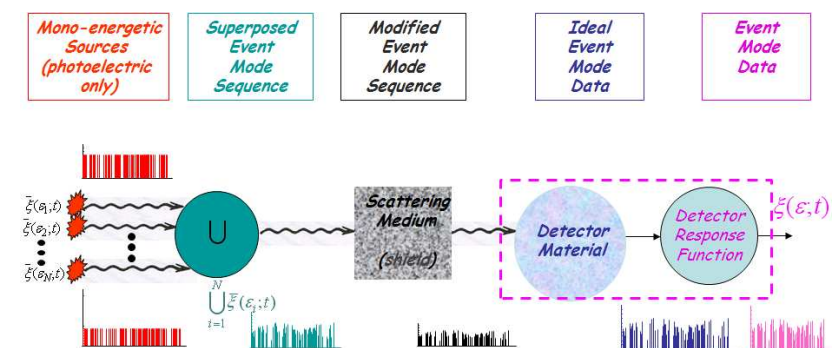

(a)

(b)

(c)

(d)

(e)

Fig. 1. Gamma-ray transport model: (a) Monoenergetic source. (b) Ideal radionuclide event mode sequence: source union/superposition. (c) Scattering medium transported EMS. (d) Detector material interaction/response. (e) Measured EMS sequence.

\section{PHYSICS-BASED PROCESSING MODEL}

In this section we discuss physics-based representations that will be incorporated into the model-based signal processors for detection and identification. The measured data consists of a low energy count, random, impulsive-like, time series measurements (energy vs time) in the form of an event mode sequence (EMS) obtained from the detector electronics [2]. A particular radionuclide can be uniquely characterized by two basic parameters: its energy and the relative intensity of $\gamma$-rays emitted [2]. Mathematically, we define the pair, $\left[\left\{\epsilon_{m \ell}\right\},\left\{\lambda_{m \ell}\right\}\right]$, as the respective energy $(\mathrm{MeV})$ and photon detection rate of the $\ell$ th-downscatter and $m$ th $\gamma$-ray line of the radionuclide.

The $\gamma$-ray is transported through a medium and interacts with materials, shield and detector resulting in the output of a list of events consisting of an event time an amplitude of pulse height or energy. It is important to realize that in the diagram of Fig. 1, we model the source radionuclide by its energy and arrival times. Since this representation of the source radionuclide contains the constituent energy and timing, then all of the information is captured by the sets, $\left[\left\{\epsilon_{m \ell}\right\},\left\{\tau_{m \ell}\right\}\right], \ell=0, \cdots, L_{\epsilon}(m), m=1, \cdots, M_{\epsilon}$.

For sequential detection we process each event in the EMS individually using a model with parameters that are analogous to the features in a pulse-height spectrum (PHS) of counts versus energy. Since a radionuclide emits $\gamma$-rays at specific energies and rates, the sequence of emitted photons can be characterized by sets of measured energies and arrival times at the detector: $\left[\left\{\epsilon_{m \ell}\right\},\left\{\tau_{m \ell}\right\}\right], \quad \ell=0, \cdots, L_{\epsilon}(m), m=$ $1, \cdots, M_{\epsilon}$. The index $m$ represents the $m$ th $\gamma$-ray line (energy) for a radionuclide with $M_{\epsilon}$ lines and the index $\ell>1$ represents the corresponding $\ell$ th "downscattered" line. It is convenient to think of each line as a separate source $(\ell=0)$. Then the emission from a given radionuclide is represented as a superposition of individual $\gamma$-ray sources. We will refer to this as the monoenergetic decomposition of the EMS for the radionuclide.

\section{A. Event Mode Sequence}

A detailed mathematical representation of the event mode sequence in terms of its monoenergetic decomposition follows directly from Refr. [10] where we extend the decomposition model to include "downscattered" photons. Using this characterization, we have the basic signal processing model in terms of the processes that govern its evolution. We start with a single $\gamma$-ray arrival which can (in our problem) be either an absorbed photon that exchanges all of its energy with an electron (photoelectric absorption) or a scattered photon that exchanges part of its energy with an electron (Compton scattering). In either case, we define $\xi\left(n ; \epsilon_{m \ell}, \tau_{m \ell}\right)$, a component of an EMS sequence, as the $n$th measured photon arrival from the $m$ th monoenergetic source of energy $(\ell=0)$ or the $\ell$ th downscatter $(\ell>1)$ of energy $\epsilon_{m \ell}(n)$, at arrival time, $\tau_{m \ell}(n)$ with associated detection rate, $\lambda_{m \ell}(n)$. The finite resolution of the detector introduces a random component to the measured energy; therefore, the energy is more accurately represented as a random variable at the $n$th arrival $\epsilon_{m \ell}(n)$. The resulting representation for a single photon arrival is $\xi\left(n ; \epsilon_{m \ell}, \tau_{m \ell}\right)=\epsilon_{m \ell}(n) \delta\left(t-\tau_{m \ell}(n)\right)$. By photon arrival we refer only to the arrival of photons that are measured by the detector and contribute to the EMS output of the detector.

In order to define the entire emission sequence over a specified observation time interval, $\left[t_{o}, T\right)$, we introduce the set notation, $\tilde{\tau}_{m \ell}:=\left\{\tau_{m \ell}(1) \cdots \tau_{m \ell}\left(N_{\epsilon}(m)\right)\right\}$ and $\tilde{\epsilon}_{m \ell}:=$ $\left\{\epsilon_{m \ell}(1) \cdots \epsilon_{m \ell}\left(N_{\epsilon}(m)\right)\right\}$ for $; \ell=0,1, \cdots, L_{\epsilon}(m) ; m=$ $0, \cdots, M_{\epsilon}$ with $N_{\epsilon}(m)$ along with $L_{\epsilon}(m)$ as the total number of counts and downscatterers for the $m$ th-source in the interval. Therefore, $\xi\left(n ; \tilde{\epsilon}_{m \ell}, \tilde{\tau}_{m \ell}\right)$ results in an impulse train of random energies from the $m \ell$ th source up to the $n$th arrival.

The interarrival time is defined by $\triangle \tau_{m \ell}(n)=\tau_{m \ell}(n)-$ $\tau_{m \ell}(n-1)$ for $\triangle \tau_{m \ell}(0)=t_{o}$ with the corresponding set definition (above) of $\triangle \tilde{\tau}_{m \ell}(n)$. Using this definition, we can rewrite with a slight abuse of notation, the photon arrival as $\xi\left(n ; \epsilon_{m \ell}, \tau_{m \ell}\right) \Rightarrow \xi\left(n ; \epsilon_{m \ell}, \triangle \tau_{m \ell}\right)=\epsilon_{m \ell}(n) \delta\left(t-\tau_{m \ell}(n)\right)$, where it is understood that $\tau_{m \ell}(n)=\tau_{m \ell}(n-1)+\triangle \tau_{m \ell}(n)$. Thus, we can rewrite the EMS in terms of interarrivals just as easily as arrivals, that is, the $m$ th monoenergetic source representation of a radionuclide characterized by its unique set of energy/arrival pairs is given by

$$
\xi\left(N_{\epsilon}(m) ; \tilde{\epsilon}_{m \ell}, \triangle \tilde{\tau}_{m \ell}\right)=\sum_{\ell=0}^{L_{\epsilon}(m)} \sum_{n=1}^{N_{\epsilon}(m)} \epsilon_{m \ell}(n) \delta\left(t-\triangle \tau_{m \ell}(n)\right)
$$

at detection rate $\lambda_{m \ell}(n)$ for $t_{o}$ known.

Following Refr. [10] this EMS model can be extended from a single monoenergetic source representation to incorporate a set of $M_{\epsilon}$-monoenergetic source components that compose a complete source radionuclide $(R N)$. such that

$$
\mathcal{R}(N ; \underline{\epsilon}, \triangle \underline{\tau})=\sum_{m=1}^{M_{\epsilon}} \xi\left(n ; \epsilon_{m \ell}(n), \triangle \tau_{m \ell}(n)\right)
$$




$$
=\sum_{m=1}^{M_{\epsilon}} \sum_{\ell=0}^{L_{\epsilon}(m)} \sum_{n=1}^{N_{\epsilon}(m)} \epsilon_{m \ell}(n) \delta\left(t-\tau_{m \ell}(n)\right)
$$

The "extended" monoenergetic decomposition includes both photoelectric and downscattered photons. The emission of photons follows a well-defined probability structure, that is, since only one photon is emitted for each event there is a fixed probability (absolute intensity $\alpha_{m \ell}$ ) that the photon is emitted with energy $\epsilon_{m \ell}$ out of $L_{\epsilon} M_{\epsilon}$ possibilities. The probabilities for a given radionuclide are specified in its energy decay diagram [2], [3]. Therefore, we model this decay structure by incorporating an indicator function defined by [12], [13]:

$$
\mathcal{I}_{j k}(m, \ell)=\left\{\begin{array}{cc}
1 & m=j \& \ell=k \\
0 & m \neq j \oplus \ell \neq k
\end{array}\right.
$$

where $\mathcal{I}_{j k}(m, \ell)$ is a random variable such that $\operatorname{Pr}\left(\mathcal{I}_{j k}(m, \ell)=\right.$ $1 \mid \xi(n ; \underline{\epsilon}, \underline{\tau}))=\operatorname{Pr}\left(\mathcal{I}_{j k}(m, \ell)=1 \mid \Xi_{n}\right)=\alpha_{j k}$ for $\alpha_{j k}$ the corresponding absolute intensity emission/occurrence probability of the $j$ th-monoenergetic radionuclide component and the $k$ th downscatter. Using the indicator function we can write the $j k$ th arrival $(m \rightarrow j ; \ell \rightarrow k$ ) of the EMS as

$$
\begin{aligned}
& \mathcal{R}(N ; \underline{\epsilon}, \triangle \underline{\tau})= \\
& \sum_{m=1}^{M_{\epsilon}} \sum_{\ell=0}^{L_{\epsilon}(m)} \sum_{n=1}^{N_{\epsilon}(m)} \mathcal{I}_{j k}(m, \ell) \epsilon_{m \ell}(n) \delta\left(t-\tau_{m \ell}(n)\right)
\end{aligned}
$$

for $\underline{\epsilon}:=\left\{\tilde{\epsilon}_{1 \ell}, \cdots, \tilde{\epsilon}_{M_{\epsilon} \ell}\right\}$, the complete set of energies composing $\mathcal{R}$ along with $\triangle \underline{\tau}:=\left\{\triangle \tilde{\tau}_{1 \ell}, \cdots, \tilde{\tau}_{M_{\epsilon} \ell}\right\}$, the corresponding set of interarrival times. The arrival index $N$ is the least upper bound of the set $N_{\epsilon}(m)$. We model the energy variations as Gaussian, $\epsilon \sim \mathcal{N}\left(\bar{\epsilon}_{m \ell}, \sigma_{\epsilon_{m \ell}}^{2}\right)$, the interarrivals as exponential, $\triangle \tau_{m \ell} \sim \mathcal{E}\left(\alpha_{m \ell} \lambda_{\triangle \tau_{m \ell}} \triangle \tau_{m \ell}(n)\right)$ [2] with the emission/occurrence probability as $\alpha_{m \ell}$.

\section{B. Compton Scattering Processing Model}

In this section we briefly discuss the simple $\gamma$-ray transport model to capture and discriminate downscattered photons. The fundamental idea is to represent the EMS as a marked Poisson process [7] specified by its rate parameter $\lambda(\epsilon)$ as a function of energy. Here the objective is to relate the source rate through the transport chain to the rate measured at the radiation detector.

The simplified $\gamma$-ray transport model developed for the model-based sequential processor is based on a simple onedimensional geometry describing the source radionuclide, shield effects and radiation detector including its material response function. The model incorporates the physics of photoelectric absorption and Compton scattering represented by the rate distributions. The general approach is to characterize the transport physics that incorporates the probabilities that the photon: (1) will escape from the material; (2) will downscatter to a lower energy; or (3) will be absorbed producing a photoelectron. Here the probability is a characteristic of both geometry and material composition. The resulting signal processing transport (SPT) model describes a fixed, onedimensional geometry of the source-shield-detector transport

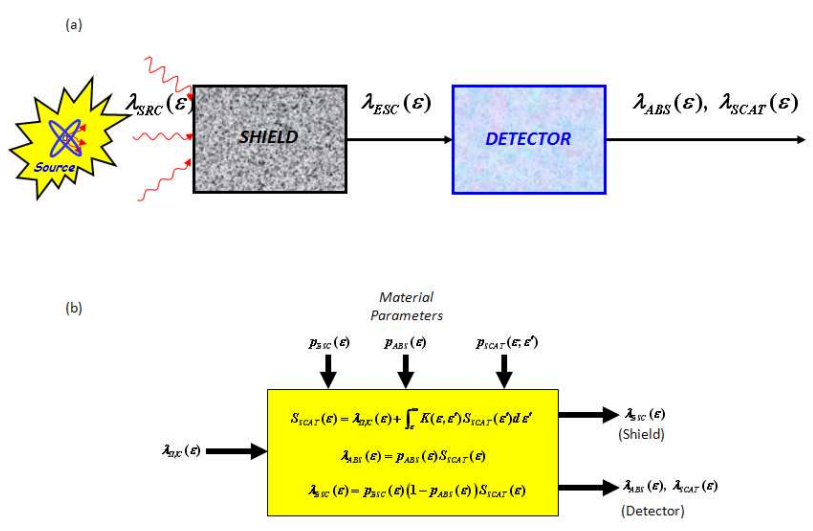

Fig. 2. Signal processing transport (SPT) model: (a) $\gamma$-ray (photon) transport chain from source to shield and detector in terms of rate probabilities. (b) SPT model fundamental transport relations.

path assuming uncorrelated photon interaction ignoring the pair production physics prevalent at higher energy $(\epsilon>$ $1 \mathrm{MeV}$ ) [2].

The basis of this approach is the mathematical projection of the 6-dimensional Boltzmann radiation transport equation to a single dimensional point-to-point equivalent for a specific geometry incorporating shielding and the detector material response. Details of the model development can be found in Chambers [9].

The SPT model is represented by the $R N$ source, shield and detector with the output rate probability distributions defined by $\lambda_{S R C}(\epsilon), \lambda_{E S C}(\epsilon), \lambda_{D E T}(\epsilon)$, respectively. The source photon emission rate distribution corresponds to the distribution of photons incident (input) to either the shield or detector, $\lambda_{I N C}(\epsilon)$, depending on the transport configuration. A fraction or probability of the incident photons will be absorbed, $p_{A B S}(\epsilon)$ with corresponding rate distribution, $\lambda_{A B S}(\epsilon)$ given by: $\lambda_{A B S}(\epsilon)=p_{A B S}(\epsilon) \times \lambda_{I N C}(\epsilon)$ while the remaining photons escape or are scattered according to $\left(1-p_{A B S}\right) \times \lambda_{I N C}(\epsilon)$. Defining the escape probability as $p_{E S C}(\epsilon)$ enables us to write the corresponding scattering rate distribution as $\lambda_{E S C}(\epsilon)$ (see Fig. 2).

To be more precise let $p_{S C A T}\left(\epsilon ; \epsilon^{\prime}\right)$ be the probability of photons at energy $\epsilon^{\prime}$ downscattered to $\epsilon$ by a single interaction with the material. The corresponding Compton scattered rate distribution can be expressed as

$$
\begin{gathered}
\lambda_{S C A T}^{(1)}(\epsilon)=\int_{\epsilon}^{\infty} p_{C O M P}\left(\epsilon ; \epsilon^{\prime}\right) \times\left(1-p_{A B S}\left(\epsilon^{\prime}\right)\right) \\
\times\left(1-p_{E S C}\left(\epsilon^{\prime}\right)\right) \times \lambda_{I N C}\left(\epsilon^{\prime}\right) d \epsilon^{\prime} \\
\lambda_{S C A T}^{(1)}(\epsilon)=\mathcal{K}\left(\epsilon ; \epsilon^{\prime}\right) \circ \lambda_{I N C}\left(\epsilon^{\prime}\right)=\int_{\epsilon}^{\infty} \mathcal{K}\left(\epsilon ; \epsilon^{\prime}\right) \lambda_{I N C}\left(\epsilon^{\prime}\right) d \epsilon^{\prime}
\end{gathered}
$$

for $p_{C O M P}\left(\epsilon ; \epsilon^{\prime}\right):=p_{S C A T}\left(\epsilon-\epsilon^{\prime} ; \epsilon^{\prime}\right)$ and the superscript $(\cdot)$ is the scattering order. This expression can be simplified further by first defining the integral operator $\mathcal{K}\left(\epsilon ; \epsilon^{\prime}\right)$ such that Eq. 4 becomes

where $\mathcal{K}\left(\epsilon ; \epsilon^{\prime}\right):=p_{S C A T}\left(\epsilon ; \epsilon^{\prime}\right) \times\left(1-p_{A B S}\left(\epsilon^{\prime}\right)\right) \times(1-$ $\left.p_{E S C}\left(\epsilon^{\prime}\right)\right)$. 
The scattered photon undergoes the same physics transport in that it can escape, be absorbed or scattered again. Therefore, for the $k$ th-order scattering, we can write the photon transport relations as

$$
\begin{aligned}
\lambda_{A B S}(k ; \epsilon) & =p_{A B S}(\epsilon) \times \lambda_{S C A T}(k-1 ; \epsilon) \\
\lambda_{E S C}(k ; \epsilon) & =p_{E S C}(\epsilon) \times\left(1-p_{A B S}(\epsilon)\right) \lambda_{S C A T}(k-1 ; \epsilon) \\
\lambda_{S C A T}(k ; \epsilon) & =\mathcal{K}\left(\epsilon ; \epsilon^{\prime}\right) \circ \lambda_{S C A T}(k-1 ; \epsilon) \\
& =\mathcal{K}^{k}\left(\epsilon ; \epsilon^{\prime}\right) \circ \lambda_{I N C}(k-1 ; \epsilon)
\end{aligned}
$$

The total rate distributions of escaped and absorbed photons are obtained by summing over all $k$-scattering orders. That is, define the total scattering function as:

$$
\begin{aligned}
\mathcal{S}_{S C A T}(\epsilon) & =\lambda_{I N C}(\epsilon)+\sum_{k=1}^{\infty} \lambda_{S C A T}(\epsilon ; k) \\
& =\left(\sum_{k=0}^{\infty} \mathcal{K}^{k}\left(\epsilon, \epsilon^{\prime}\right)\right) \circ \lambda_{I N C}(\epsilon)
\end{aligned}
$$

or since the sum is the Neumann series solution of the operator equation, we can write

$$
\mathcal{S}_{S C A T}(\epsilon)=\lambda_{I N C}(\epsilon)+\int_{\epsilon}^{\infty} \mathcal{K}\left(\epsilon, \epsilon^{\prime}\right) \mathcal{S}_{S C A T}\left(\epsilon^{\prime}\right) d \epsilon^{\prime}
$$

which leads to the modified transport relations (see Fig. 2b):

$$
\begin{aligned}
\lambda_{A B S}(\epsilon)= & p_{A B S}(\epsilon) \times \mathcal{S}_{S C A T}(\epsilon) \\
\lambda_{E S C}(\epsilon)= & p_{E S C}(\epsilon) \times\left(1-p_{A B S}(\epsilon)\right) \mathcal{S}_{S C A T}(\epsilon) \\
\lambda_{S C A T}(\epsilon)= & \int_{\epsilon}^{\infty} p_{S C A T}\left(\epsilon ; \epsilon^{\prime}\right) \times\left(1-p_{A B S}\left(\epsilon^{\prime}\right)\right) \\
& \times\left(1-p_{E S C}\left(\epsilon^{\prime}\right)\right) \mathcal{S}_{S C A T}\left(\epsilon^{\prime}\right) d \epsilon^{\prime}
\end{aligned}
$$

Solving these equations enable us to estimate the rate distributions of both absorbed and escaped photons for all orders of scattering. The model has been developed and validated using sophisticated Monte Carlo simulation algorithms (see [9] for more details).

From the signal processing perspective, we know that the sequential decision function (to follow) requires that for each photon arrival the energy, detection rate (reciprocal mean interarrival time) and emission/occurrence (photoelectric/downscatter) probabilities must be estimated. Since both photoelectric and downscattered arrivals can have the same energy but can have different monoenergetic target sources, it is the "scattering (occurrence) probability" or equivalently the emission probability (for photoelectrons) that must be estimated. These parameters act as inherent weighting functions and therefore each arrival can be thought of as being partitioned according to the various weights into the composite $R N$ decision function to make the decision.

\section{RADIONUCLIDE DETECTION}

The development of a radionuclide detector based on photon-by-photon processing follows. Thus, we develop a "sequential" technique that is aimed at processing a single

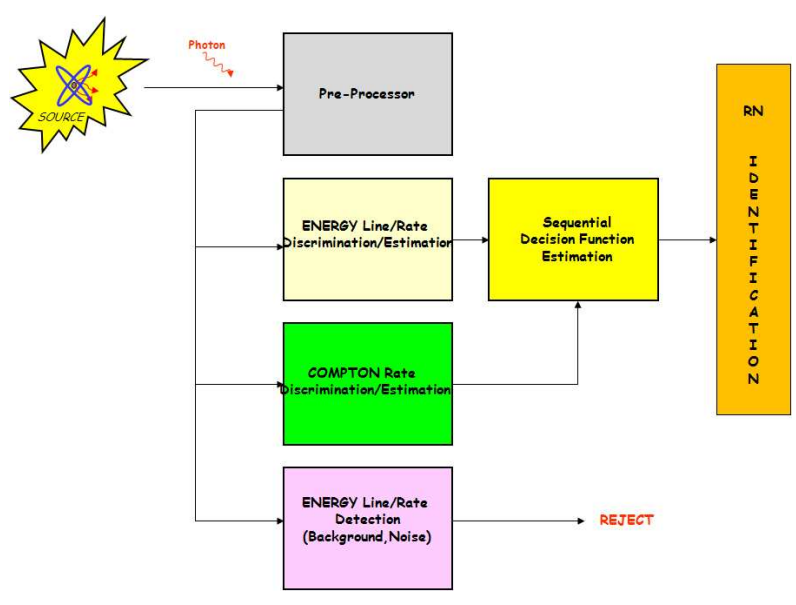

Fig. 3. Bayesian radiation detection: Acquisition, pre-processing (optional), energy/rate discrimination/estimation, Compton rate discrimination/estimation, background and extraneous line rejection, decision function estimation and $R N$ identification.

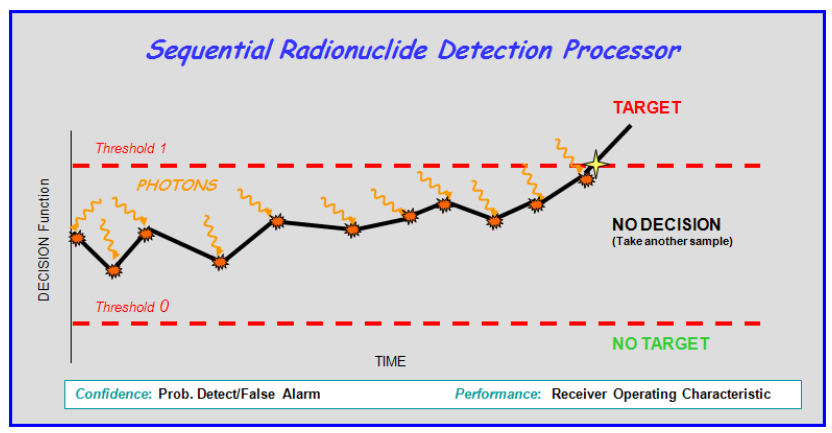

Fig. 4. Conceptual implementation (operation) of the sequential Bayesian radionuclide detection technique. As each individual photon is extracted, it is discriminated, estimated, the decision function (log-likelihood) calculated and compared to a threshold to "decide" if the targeted radionuclide is present or not. Quantitative performance and sequential thresholds are determined from an estimated ROC curve by selecting an operating point (detection/false alarm probability).

photon arrival at a time rather than attempting to perform a batch solution which is the basis of PHS analysis [2]. The basic approach is shown in Fig. 3. After a single photon is preprocessed by the measurement system, both energy and arrival time are extracted and passed onto energy/rate discriminators to determine whether or not it is a targeted photon or its downscatter. If acceptable (target), parameter estimates are sequentially updated and provided as input to the decision function for detection and identification. If not acceptable, the photon is rejected and discarded (noise or background). Conceptually, we illustrate the sequential detector operation in Fig. 4 showing each photon arrival along with the corresponding decision function and thresholds.

To formally pose the radionuclide detection problem, we appeal to classical (sequential) detection theory [14]. We are to test the binary hypothesis that the measured EMS has evolved from the targeted radionuclide characterized uniquely from its monoenergetic decomposition of Eq. 3. Therefore, we specify 
the hypothesis test

$$
\begin{aligned}
& \mathcal{H}_{0}: \xi(n ; \underline{\epsilon}, \triangle \underline{\tau})=\mathcal{R}(n ; \underline{\epsilon}, \triangle \underline{\tau})+\nu(n) \quad[\text { NON-TARGET } \\
& \mathcal{H}_{1}: \xi(n ; \underline{\epsilon}, \triangle \underline{\tau})=\mathcal{R}\left(n ; \underline{\epsilon}^{t}, \triangle \underline{\tau}^{t}\right)+\nu(n) \quad[\text { TARGET] }
\end{aligned}
$$

where $\mathcal{R}(n ; \underline{\epsilon}, \triangle \underline{\tau})$ is the random composite EMS of Eq. 3 contaminated with zero-mean, Gaussian measurement (instrumentation) noise, $\nu \sim \mathcal{N}\left(0, \sigma_{\nu}^{2}\right)$ for $\epsilon_{m \ell} \sim \mathcal{N}\left(\bar{\epsilon}_{m \ell}, \sigma_{\epsilon_{m \ell}}^{2}\right)$ and $\triangle \tau_{m \ell} \sim \mathcal{E}\left(\lambda_{\triangle \tau_{m \ell}} \triangle \tau_{m \ell}(n)\right)$. Here the superscript ${ }^{t}$ is used to denote the "true" or "target" parameters.

The optimal solution to this binary decision problem is based on applying the Neyman-Pearson theorem leading to the likelihood given by the ratio of probabilities [14]-[16]. However, since the distributions under investigation are members of the exponential family [12], then taking logarithms leads to the sequential log-likelihood ratio

$$
\begin{aligned}
\Lambda\left[\Xi_{n}\right] & =\Lambda\left[\Xi_{n-1}\right]+\ln \operatorname{Pr}\left(\xi(n ; \underline{\epsilon}, \triangle \underline{\tau}) \mid \Xi_{n-1}, \mathcal{H}_{1}\right) \\
& -\ln \operatorname{Pr}\left(\xi(n ; \underline{\epsilon}, \triangle \underline{\tau}) \mid \Xi_{n-1}, \mathcal{H}_{0}\right)
\end{aligned}
$$

and therefore, the Wald sequential probability-ratio test becomes [15], [16] (see Fig. 3)

$$
\begin{array}{clrl}
\Lambda\left[\Xi_{n}\right] & \geq \ln \mathcal{T}_{1}(n) & \text { Accept } \mathcal{H}_{1} \\
\ln \mathcal{T}_{0}(n) \leq \Lambda\left[\Xi_{n}\right] & \leq \ln \mathcal{T}_{1}(n) & \text { Continue } \\
\Lambda\left[\Xi_{n}\right] & \leq \ln \mathcal{T}_{0}(n) & \text { Accept } \mathcal{H}_{0}
\end{array}
$$

where the set of EMS measurements are $\Xi_{N}:=$ $\{\xi(0), \xi(1), \cdots, \xi(N)\}$ and the thresholds are specified in terms of the false alarm $\left(\mathrm{P}_{F A}\right)$ and miss $\left(\mathrm{P}_{M}\right)$ probabilities as

$$
\mathcal{T}_{0}(n)=\frac{\mathrm{P}_{M}(n)}{\mathrm{P}_{F A}(n)} \quad \mathcal{T}_{1}(n)=\frac{1-\mathrm{P}_{M}(n)}{\mathrm{P}_{F A}(n)}
$$

typically obtained by generating receiver operating characteristic $(R O C)$ curves and selecting an operating point $\left(P_{F A}, P_{M}\right)$. So we see that at each photon arrival (at time $n$ ), we can sequentially update the likelihood and thresholds to perform the detection - "photon-by-photon."

To implement this detection technique (see Eq. 9), we must specify the required distributions in order to calculate the decision function It can be shown that [11] the sequential loglikelihood ratio detector is given by

$$
\begin{aligned}
& \Lambda\left[\Xi_{n}\right]=\Lambda\left[\Xi_{n-1}\right]+ \\
& \sum_{m=1}^{M_{\epsilon}} \sum_{\ell=0}^{L_{\epsilon}(m)} \ln \Theta_{m \ell}\left(n ; \theta, \mathcal{H}_{1}\right)-\sum_{m=1}^{M_{\epsilon}} \sum_{\ell=0}^{L_{\epsilon}(m)} \ln \Theta_{m \ell}\left(n ; \theta, \mathcal{H}_{0}\right)
\end{aligned}
$$

where

$$
\begin{aligned}
& \Theta_{m \ell}\left(n ; \theta, \mathcal{H}_{i}\right):= \\
& \alpha_{m \ell} \times \operatorname{Pr}\left(\triangle \tau_{m \ell}(n) \mid \epsilon_{m \ell}(n), \mathcal{I}_{j k}(m, \ell), \Xi_{n-1}, \mathcal{H}_{i}\right) \times \\
& \operatorname{Pr}\left(\epsilon_{m \ell}(n) \mid \mathcal{I}_{j k}(m, \ell), \Xi_{n-1}, \mathcal{H}_{i}\right) \\
& \text { and recall the emission/occurrence probability is } \\
& \alpha_{m \ell}=\operatorname{Pr}\left(\mathcal{I}_{j k}(m, \ell) \mid \Xi_{n-1}, \mathcal{H}_{i}\right) ; \quad i=0,1
\end{aligned}
$$

giving us the general form required for our problem. Note that this formulation provides us with a channel-by-channel (photon-by-photon) processor, since the $m \ell$-th terms are available at the output of each channel.

It can also be shown [11] that by defining the photoelectric (absorption) log-likelihood as $\Lambda_{p e}\left[\Xi_{n-1}\right]$ and the downscatter log-likelihood as $\Lambda_{d s}\left[\Xi_{n-1}\right]$, we obtain the decomposition leading to the algorithmic structure (see Fig. 5)

$$
\Lambda\left[\Xi_{n}\right]=\Lambda\left[\Xi_{n-1}\right]+\Lambda_{p e}\left[\Xi_{n-1}\right]+\Lambda_{d s}\left[\Xi_{n-1}\right]
$$

where

$$
\begin{aligned}
\Lambda_{p e}\left[\Xi_{n-1}\right]:= & \sum_{m=1}^{M_{\epsilon}} \ln \Theta_{m 0}\left(n ; \theta, \mathcal{H}_{1}\right)-\ln \Theta_{m 0}\left(n ; \theta, \mathcal{H}_{0}\right) \\
\Lambda_{d s}\left[\Xi_{n-1}\right]:= & \sum_{m=1}^{M_{\epsilon}} \sum_{\ell=1}^{L_{\epsilon}(m)} \ln \Theta_{m \ell}\left(n ; \theta, \mathcal{H}_{1}\right) \\
& -\ln \Theta_{m \ell}\left(n ; \theta, \mathcal{H}_{0}\right)
\end{aligned}
$$

Not surprisingly, we see that this log-likelihood decomposition shows that if we ignore the downscattered photons as in Refr. [10] we cannot achieve the optimal decision because we are not using all of the physics information available! Thus, we expect the performance of this "extended" processor to be superior to the photoelectric only implementation!

The sequential radiation detection processor is illustrated in Fig. 5. As the photon arrives, its energy and interarrival time are extracted and discriminated to select the appropriate channel for processing. If it is a targeted photoelectron, then it is processed precisely as discussed in Refr. [10], that is, after discrimination the energy, interarrival (detection rate) and emission/occurrence probability parameters are estimated, the appropriate decision function updated and compared to the threshold to "decide" if the targeted $R N$ is present. On the other hand, if the arrival is not deemed a photoelectron, then it is discriminated to investigate the possibility of it being a potential downscattered arrival. Here discrimination is based on the selected energies associated with the target radionuclide and its corresponding Compton region. That is, for example with knowledge of the target $R N$ Compton edge [2], a number $\left(L_{\epsilon}(m)\right)$ of downscattered energies or bins are selected and used to specify a set of discriminants for Compton processing. If accepted, then parameter estimation is performed to enhance the energy which is used in the SPT model to provide an estimate of the corresponding detection rate and emission/occurrence probability. Thus, all of the downscatter parameters are updated along with the corresponding "true" decision functions. It is interesting to note that we can conceive of the downscattered photon information being partitioned into a set of weights (occurrence probabilities) and updating the (partial) decision functions simultaneously as prescribed by the optimal decision function. Once this information is extracted and the appropriate decision functions updated, then the threshold is tested to decide on the presence of a targeted 


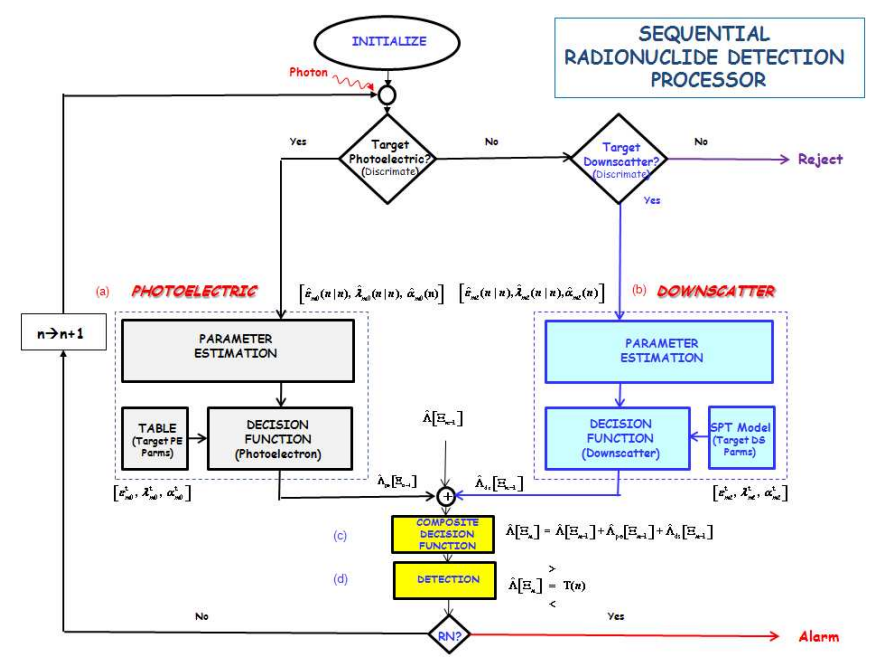

Fig. 5. Flow diagram of radionuclide detection processor: (a) Photoelectric processor. (b) Downscatter processor. (c) Decision function. (d) $R N$ Detection

$R N$ as shown in the figure. If the photon is not photoelectric, then it is rejected and the next arrival is processed.

Applying statistical models of our problem, that is, each energy component is assumed independent Gaussian and the corresponding interarrival times are exponentially distributed [2] For our problem we can re-write Eq. 12 using the corresponding parameter estimates as the final sequential loglikelihood ratio radionuclide detector. That is, we require the set of targeted (true) parameters, $\left[\left\{\underline{\alpha}^{t}\right\},\left\{\underline{\epsilon}^{t}\right\},\left\{\triangle \underline{\tau}^{t}\right\}\right]$ and estimated parameters, $[\{\underline{\hat{\alpha}}\},\{\underline{\hat{\hat{\epsilon}}}\},\{\triangle \underline{\hat{\tau}}\}]$. Once acquired, the decision function can be calculated and compared to the thresholds which have been estimated from ROC curve operating points.

$$
\begin{aligned}
& \Lambda\left[\Xi_{n}\right]=\Lambda\left[\Xi_{n-1}\right]+ \\
& \sum_{m=1}^{M_{\epsilon}} \sum_{\ell=0}^{L_{\epsilon}(m)} \ln \left(\frac{\left(\alpha_{m \ell}^{t}\right)^{2} \lambda_{\triangle \tau_{m \ell}}^{t}}{\sqrt{2 \pi} \sigma_{\epsilon_{m \ell}^{t}}}\right)-\ln \left(\frac{\hat{\alpha}_{m \ell}^{2} \hat{\lambda}_{\triangle \tau_{m \ell}}(n \mid n)}{\sqrt{2 \pi} \hat{\sigma}_{\epsilon_{m \ell}}(n \mid n)}\right) \\
& +\left(\hat{\alpha}_{m \ell} \hat{\lambda}_{\triangle \tau_{m \ell}}(n \mid n)-\alpha_{m \ell}^{t} \lambda_{\triangle \tau_{m \ell}}^{t}\right) \xi_{\triangle \tau}(n) \\
& +\frac{1}{2}\left(\frac{\xi_{\epsilon}(n)-\hat{\bar{\epsilon}}_{m \ell}(n \mid n)}{\hat{\sigma}_{\epsilon_{m \ell}}(n \mid n)}\right)^{2}-\frac{1}{2}\left(\frac{\xi_{\epsilon}(n)-\epsilon_{m \ell}^{t}(n)}{\sigma_{\epsilon_{m \ell}^{t}}}\right)^{2}
\end{aligned}
$$

The detailed structure of the "extended" sequential processor is shown in Fig. 6 with parameter estimates (to follow) in the figure replacing the parameters of Eq. 13, that is, $\hat{\epsilon}_{m \ell}(n) \rightarrow \epsilon_{m \ell}(n)$. Here we observe the underlying parallel/distributed structure as in Refr. [10] but for both the photoelectric and downscattered arrivals. Following the arguments in Refr. [10], since each individual $R N$ is uniquely specified (statistically) by its parameter set, $\{\underline{\epsilon}, \triangle \underline{\tau}, \underline{\alpha}\}$, we assume that $\underline{\alpha}$ is known for each target (from Tables [3] or from the SPT model [9]). This completes the structure of the sequential Bayesian radiation detector, we will discuss the actual photon-by-photon implementation of this processor in the next section.

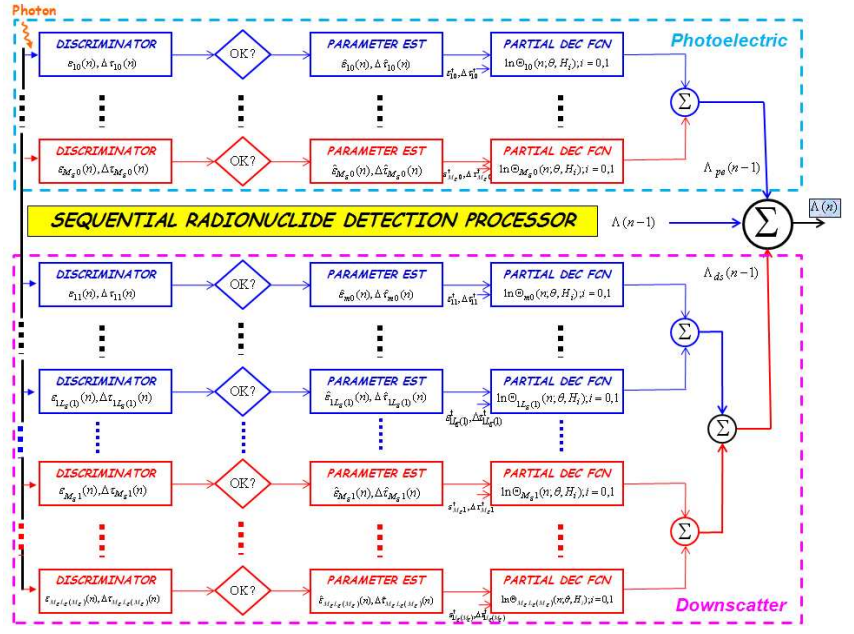

Fig. 6. Detailed parallel/distributed implementation $M B P$ structure for extended photoelectric absorption and Compton downscatter illustrating both photoelectric and downscatter discrimination, parameter estimation ( $L K F$ and $P F)$ and complete decision function (log-likelihood) estimates.

\section{IMPLEMENTATION}

There are three phases to the implementation: (1) discrimination; (2) estimation; and (3) detection as shown in Fig. 5. The first step is to discriminate the arrival to ascertain whether or not it is one of the targeted $R N$ components, that is, either a photoelectron or a downscattered photon. Discrimination is performed using truth data from Tables [3] and the output of SPT model [9] for downscatter along with the resulting parameters using calibration data to construct confidence intervals for both energy and average interarrivals. It is here that the calibrated one dimensional SPT model is applied to obtain the targeted downscatter detection rates and occurrence probabilities for the interval constructs. Once this step is accomplished and the arrival is accepted, then the parameter estimation step is performed. Here the energy, interarrival and emission/occurrence probability are estimated using the distribution models: energy/Gaussian (linear Kalman filter), interarrival/Exponential (particle filter) and emission/occurrence probability (sequential counter). With these parameter estimates available we can then calculate the decision function (detection) by incorporating both photoelectron and downscattered arrivals. The thresholds are pre-calculated from ROC curves and the operating point (detection and false alarm probability) selected.

Let us track a single photon arrival through the processing steps as our road map and illustrated in Fig. 5. The processor is partitioned into two subprocessors: one for photoelectrons and one for downscattered photons. Each channel is structurally identical only the targeted parameters are different. The idea is that for each arrival the complete log-likelihood decision function $(\Lambda(n))$ is sequentially updated until a threshold is exceeded declaring the presence or non-presence of the targeted $R N$. When the photon arrives, it is first discriminated to discern whether it is a targeted photoelectron, if so, it is processed and the appropriate decision function updated. 
If not, it is passed to the bank of downscattered channel processors and discriminated as to whether it is a target downscatter based on pre-selected acceptable rate ranges. If so, then each downscatter channel updates its corresponding decision function after parameter estimation. If not, the photon is rejected.

First, we investigate the individual channel processor one for each energy/rate composing the target radionuclide.

\section{A. Discrimination}

Based on the parallel/distributed architecture of the processor, we first apply an energy discriminator to "decide" which channel the photon should be processed followed by a detection rate discriminator using the interarrival for verification. The discriminators use implied hypothesis testing by constructing confidence intervals about the means of the respective parameters [12].

1) Energy Discriminator: The energy discriminator performs the following confidence interval test to accept or reject the photon in the $m \ell$-th channel:

$$
\left[\epsilon_{m \ell}^{t}-\kappa_{\gamma} \sigma_{\xi} \leq \epsilon_{m \ell}(n) \leq \epsilon_{m \ell}^{t}+\kappa_{\gamma} \sigma_{\xi}\right]
$$

where $\epsilon_{m \ell}^{t}$ is the true ( $m \ell$-th channel) energy associated with the targeted radionuclide, $\kappa_{\gamma}$ is the respective confidence coefficient with associated confidence level $\gamma$ and $\sigma_{\xi}$ is the standard deviation associated with the precision of the measurement.

2) Interarrival Discriminator: The interarrival measurement is tested similarly by the following confidence interval discriminator. For large $n$, the estimate of the mean interarrival time is approximately Gaussian, $\triangle \hat{\bar{\tau}} \sim \mathcal{N}\left(\triangle \tau^{t}, \triangle \tau^{t} / \sqrt{n}\right)$.

$$
\left[\triangle \tau_{m \ell}^{t}-\kappa_{\tilde{\gamma}} \sigma_{\triangle \bar{\tau}} \leq \triangle \hat{\bar{\tau}}_{m \ell}(n) \leq \triangle \tau_{m \ell}^{t}+\kappa_{\tilde{\gamma}} \sigma_{\triangle \bar{\tau}}\right]
$$

where $\triangle \tau_{m \ell}^{t}$ is the true (channel) interarrival time associated with the targeted radionuclide, $\kappa_{\tilde{\gamma}}$ is the respective confidence coefficient with associated confidence level $\tilde{\gamma}$ and $\sigma_{\triangle \bar{\tau}}$ is the standard deviation or the variance of the estimated mean interarrival time, $\triangle \hat{\bar{\tau}}_{m \ell}$. Events with interarrival times that cause the estimated mean to deviate outside the confidence bounds are rejected.

3) Photoelectric Interarrival Discriminator: For photoelectric discrimination, the mean interarrival time is not necessarily the actual source interarrival, but may include scattered or background photon interarrivals, we use an estimated PHS obtained during the calibration phase to approximate the percentage $\left(\beta_{o}\right)$ of downscattered photons to estimate $\triangle \tau^{t}$ [1],[2]. $\beta_{o}$ is based on the ratio of a fixed number of higher energy bin counts to the target energy bin count. Because of its Poisson nature, we average the interarrivals associated with the $m \ell$ th-bin taking its reciprocal to obtain the composite (total) rate, $\lambda_{\epsilon_{m 0}}$. Finally, we estimate the true detection rate as

$$
\lambda_{m 0}^{t}=\left(1-\beta_{o}\right) \lambda_{\epsilon_{m 0}}, \quad \Delta \tau^{t}=\frac{1}{\lambda_{m 0}^{t}}
$$

4) Downscatter Discriminator: For downscatter discrimination, the "true" or table parameters are obtained by using the SPT model rather than Tables directly. After calibration to scale each targeted photoelectron, the associated PHS is generated as shown in Fig. 7. A table look-up approach is employed in the processor by using the SPT model to generate a matrix of parameters: $\left[\left\{\epsilon_{m \ell}^{t}\right\},\left\{\lambda_{m \ell}^{t}\right\},\left\{\alpha_{m \ell}^{t}\right\}\right] ; \ell=$ $1, \cdots, L_{\epsilon}(m) ; m=1, \cdots, M_{\epsilon}$; In Fig. 7a, we show the partial table of energy for each targeted $\gamma$-ray line ( $m$-th), while in $7 \mathrm{~b}$, we observe a pictorial representation of the energy bins. In Fig. $7 \mathrm{c}$, we see the output of the SPT model associating each $\gamma$-ray line (photopeak) of the PHS with its corresponding (predicted) downscatter spectrum. The horizontal rectangles represent the "range" of bins selected for discrimination corresponding to the highest probability region (HPR) of the PHS. Each line has its own, pre-selected range. When a downscatter photon is discriminated, the appropriate bin in selected (vertical rectangle in d) and the corresponding "true" occurrence probabilities $\left(\alpha_{m \ell}\right)$ located in the SPT model generated table-one for each downscatter target photon residing in the selected bin. In this way, the SPT model is used to provide the "true" parameter values for decision function input.

The design constraint for the downscatter range of rates is based on the SPT model output which provides a predicted PHS obtained during the initial calibration data. Here either all arrivals can be processed or a constrained subset selected from the HPR observed in the SPT model predicted PHS. In this way, as the single downscatter photon is processed, it follows the identical steps as the photoelectron arrivals, but uses the SPT model to determine the corresponding emission/occurrence probability incorporated into the decision function.

Note that Compton-edge regions of various targeted $R N$ can overlap (see Fig. 7c) and therefore each must be simultaneously updated in its respective channels and weighted by their corresponding emission/occurrence probabilities in the decision function calculation. Thus, for a downscattered arrival, we can envision it being partitioned by weight (emission/occurrence probability) updating more than one loglikelihood decision function simultaneously.

\section{B. Detection}

Sequential radionuclide detection is implemented in a channel-by-channel framework. For a given set of radionuclides, the distributions associated with each individual monoenergetic component are calculated in parallel at each channel and then combined in the detector/identifier. The sequential radiation detector has three possible choices to discern the incoming photon arrival: (1) a target photoelectron; or (2) a target downscattered photon; or (3) a background/noise photon. If it is deemed background/noise, then the photon is rejected. However, if it is a photoelectron or downscatter, then it is processed separately. Thus, two stages of discrimination are required, rather than the single stage of Refr. [10]. First, the arrival is discriminated to be a photoelectron and processed as such, if not, it is discriminated to be a downscatter photon and 


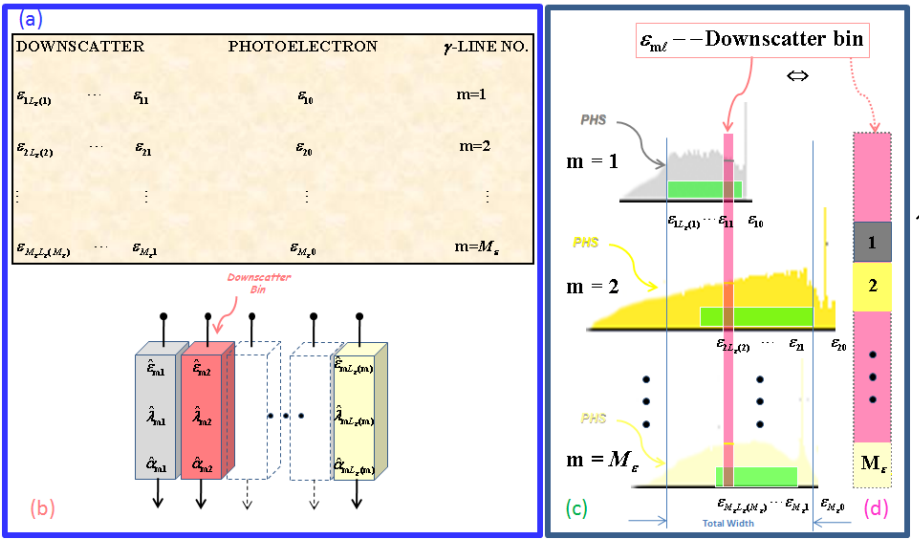

Fig. 7. Rate distribution decomposition based on SPT model: (a) Energy decomposition: downscatter $(\ell>0)$ and photoelectric $(\ell=0)$. (b) Energy bin composition: energy, rate and emission/occurrence probability parameters. (c) SPT model downscatter decomposition (PHS): Selected downscatter energy channels (horizontal rectangles (green) illustrating overlap for each ( $m$-th) PHS. (d) "Zoomed" energy bin illustrating decomposition and probability contribution of each (overlapped) downscatter photon.

then processed using a model-based scheme to extract its required parameters for detection. Once extracted the appropriate (partial) decision functions are updated accordingly. Thus, the sequential radionuclide detector is implemented in a channelby-channel framework. Basically, the individual distributions are calculated in parallel at each channel and then combined in the detector/identifier. At each arrival after discrimination, the accepted channel $j k$ th photon is processed by the energy and interarrival parameter estimators $(\hat{\theta})$ providing the input to the log-likelihood ratio decision function along with the truth parameters $\left(\theta^{t} \rightarrow\left[\left\{\epsilon_{m \ell}^{t}\right\},\left\{\triangle \tau_{m \ell}^{t}\right\},\left\{\alpha_{m \ell}^{t}\right\}\right] ; \quad \ell=\right.$ $\left.0, \cdots, L_{\epsilon}(m) ; m=1, \cdots, M_{\epsilon}\right)$ from the Tables [3] and SPT model [9].

Thus, after successful discrimination the parameters are estimated and employed to calculate the log-likelihood function. These are estimated channel-by-channel ( $m \ell$-th-channel) and the overall decision function implemented sequentially (in arrival time). Once the parameters are estimated they are implemented in each channel log-likelihood partial calculation $\left(\Theta_{m \ell}(n ; \theta)\right)$ and all of the partial sums are combined along with the previous (in arrival time) log-likelihood to sequentially update the new log-likelihood at time $n$. It is then compared to the threshold to see if a detection is possible. If not, the next photon is processed and the log-likelihood updated to see if a decision can be made. This sequential radionuclide process continues until there is enough data to justify a decision.

Table I. Sequential Radiation Detection with Scattering $\underline{\text { Discrimination }}$

$$
\begin{gathered}
{\left[\epsilon_{m \ell}^{t}-\kappa_{\gamma} \sigma_{\xi} \leq \epsilon_{m \ell}(n) \leq \epsilon_{m \ell}^{t}+\kappa_{\gamma} \sigma_{\xi}\right] \quad \text { (energy) }} \\
{\left[\triangle \tau_{m \ell}^{t}-\kappa_{\tilde{\gamma}} \sigma_{\triangle \bar{\tau}} \leq \triangle \hat{\bar{\tau}}_{m \ell}(n) \leq \triangle \tau_{m \ell}^{t}+\kappa_{\tilde{\gamma}} \sigma_{\triangle \bar{\tau}}\right] \quad \text { (interarrival) }}
\end{gathered}
$$

\section{Estimation}

$$
\begin{aligned}
\hat{\epsilon}_{m \ell}(n \mid n) & =\hat{\epsilon}_{m \ell}(n \mid n-1)+K_{\epsilon_{m \ell}} i_{m \ell}(n) \quad \text { (energy) } \\
\triangle \hat{\tau}_{m \ell}(n \mid n) & =\arg \max \hat{\operatorname{Pr}}\left(\triangle \tau_{m \ell}(n) \mid \Xi_{n}\right) \quad \text { (interarrival) } \\
\hat{\lambda}_{\triangle \tau_{m \ell}}(n \mid n) & =\frac{1}{\triangle \hat{\tau}_{m \ell}(n \mid n)} \quad \text { (detection rate) } \\
\hat{\alpha}_{m \ell}(n) & =\frac{N_{\epsilon_{m \ell}}(n)}{M_{\epsilon}(n)} \quad(\text { emission probability) } \\
\Lambda\left[\Xi_{n}\right] & =\Lambda\left[\Xi_{n-1}\right]+\ln \Theta_{m \ell}\left(n ; \theta^{t}\right)-\ln \Theta_{m \ell}(n ; \hat{\theta})(\mathrm{dcn})
\end{aligned}
$$

$\underline{\text { Detection }}$

$$
\begin{array}{cccc} 
& \mathcal{H}_{1} & & \\
& \geq & \mathcal{T}_{1} & \\
& = & \text { Continue } & \text { (log-likelihood) } \\
& \leq & \left.\mathcal{T}_{0}\right] & \\
& \Xi_{0} & &
\end{array}
$$

\section{RESUlts}

A proof-of-concept experiment was developed [10] to assess the feasibility of the sequential Bayesian processor. Three source radionuclides (cobalt $\left({ }^{60} \mathrm{Co}\right)$, cesium $\left({ }^{137} \mathrm{Cs}\right)$, barium $\left({ }^{133} \mathrm{Ba}\right)$ ) were targeted in a laboratory environment contaminated with background and extraneous sources that were stored in surrounding cabinets. The equipment used in the experiment consisting of sources, measurement instruments including a HPGe commercial detector. The sources were positioned such that they were centered on a direct line with the HPGe detector face at a distance of $100 \mathrm{~cm}$ for $1000 \mathrm{sec}$. Each target source and background was individually counted with the results combined to generate the controlled "feasibility" data set. The primary objective was to assess the feasibility of the processor along with ability to detect and classify targeted radionuclides.

The sequential Bayesian detector was applied to the "feasibility" data set. a set of experimental composite radionuclide EMS data consisting of three radionuclides cobalt $\left({ }^{60} \mathrm{Co}\right)$, cesium $\left({ }^{137} \mathrm{Cs}\right)$, barium $\left({ }^{133} \mathrm{Ba}\right)$ with 2,1 and 5 energy lines (monoenergetic sources), respectively along with background and an extraneous potassium source. After an initial calibration phase of the algorithm which consisted of "tuning" the Bayesian processors on simulated and controlled data, setting initial parameters, etc., the overall results of the processing are shown in Fig. 8. We note three columns of data, the first column is the composite pulse-height spectrum, with the second the composite EMS with the circles representing the discriminator output photons. The final column is the log-likelihood decision functions for each of the targeted radionuclides.

As each photon is processed, the decision function is sequentially updated until one of the thresholds (target/nontarget) is crossed (lighter crosses in figure) declaring a threat or non-threat. The results of the photoelectric and downscatter processor are shown in Fig. 8 where we observe detection times of 6.6 secs (PE: 7.1 secs), 0.7 secs (PE: 1.0 secs), and 0.53 secs (PE: 0.85 secs) respectively for the cobalt, cesium and barium radionuclides. This performance is expected since the "new" downscatter photon information is incorporated into 


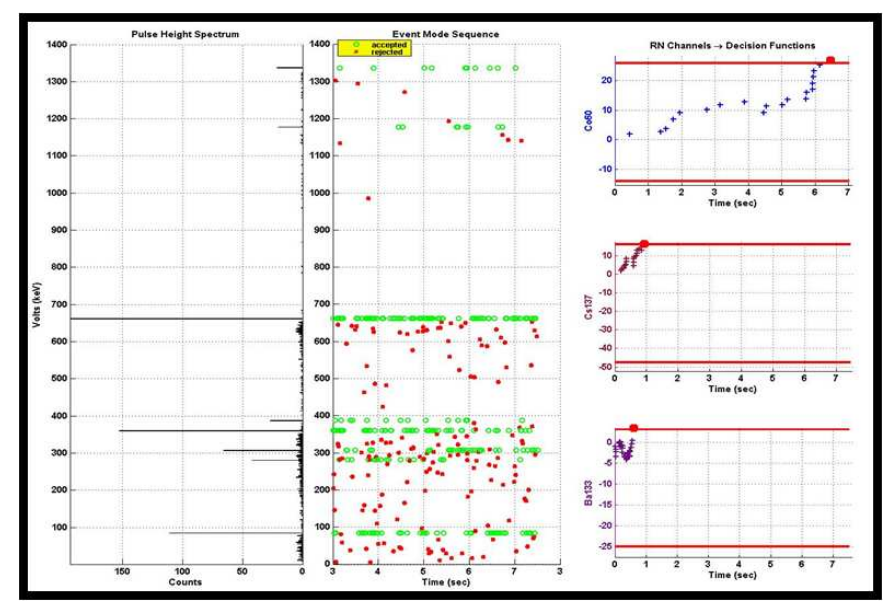

Fig. 8. Photoelectric and downscatter Bayesian detection and identification. (a) Pulse-height spectrum (after calibration). (b) EMS with discrimination (circles). (c) Log-likelihood decision functions for ${ }^{60} \mathrm{Co}$ (detection time: $4.05 \mathrm{sec}$ ), ${ }^{137} \mathrm{Cs}$ (detection time: $0.678 \mathrm{sec}$ ) and ${ }^{133} \mathrm{Ba}$ (detection time: $0.513 \mathrm{sec}$ ) radionuclide detection/identification.

each channel providing a more timely detection time than the basic photoelectric (PE) only processor.

It should also be noted that the thresholds are determined from a receiver operating characteristic (ROC) curve for each radionuclide decision function. That is, we synthesize EMS and noise sequences using a brute force approach to estimate the $R O C$ curves in order to calculate the required thresholds. Based on the experimental $S N R$, the selected detection and false-alarm probabilities were $(98 \%, 2 \%)$ specifying the thresholds corresponding to this ROC operating point and calculated according to Eq. 11 for each radionuclide.

Thus, we have shown that a sequential Bayesian detection processor can be developed to provide a feasible solution to the radiation detection problem. This was achieved by defining a target radionuclide(s) and its monoenergetic decomposition including downscatter evolving from the underlying transport physics of the photon and measurement process. The key idea was to process the data, photon-by-photon, rejecting any extraneous non-targeted radionuclide measurements and processing only those that correspond to the targeted threat radionuclide(s) while identifying downscattered photons and extracting the underlying information for processing. The new extended processor performed quite well.

Here we "extended" this model to incorporate downscatter physics (Compton) and developed the corresponding Bayesian probabilistic framework to theoretically define the problem. A particular realization of the process was successfully developed and applied to proof-of-concept experimental data demonstrating its overall feasibility. The key idea was to process the data (as before [10]), photon-by-photon, rejecting any extraneous non-targeted radionuclide measurements and processing only those that correspond to the targeted threat radionuclide(s) while identifying downscattered photons and extracting the underlying information for processing. The new extended processor performed quite well compared to the previous "photoelectric only" design of our previous work [10].

\section{ACKNOWLEDGMENTS}

We would like to acknowledge the contributions of our nuclear experts, Dr. T. Gosnell, Dr. D. Slaughter, Professor S. Prussin, and Mr. D. Manatt for their encouragement, guidance and tremendous insight in helping to direct our efforts. This work performed under the auspices of the U.S. Department of Energy by Lawrence Livermore National Laboratory under Contract DE-AC52-07NA27344.

\section{REFERENCES}

[1] R. D. Evans, The Atomic Nucleus. New York, New York: McGraw-Hill, 1985.

[2] G. F. Knoll, Radiation Detection and Measurement, 3rd ed., Hoboken New Jersey: John Wiley, 2000.

[3] M. Herman, Dir., National Nuclear Data Center, Brookhaven Nat. Lab., Available: www.nndc.bnl.gov, 1986.

[4] L. J. Meng and D. Ramsden, An inter-comparison of three spectraldeconvolution algorithms for gamma-ray spectroscopy, IEEE Trans. Nucl. Sci., Vol. 47, no. 4, pp. 1329-1336, 2000.

[5] J. V. Candy, Model-Based Signal Processing, Hoboken, New Jersey: John Wiley/IEEE Press, 2006.

[6] J. V. Candy, Bayesian Signal Processing, Classical, Modern and Particle Filtering, Hoboken New Jersey: John Wiley/IEEE Press, 2009.

[7] D. L. Snyder and M. I. Miller, Random Point Process in Time and Space. New York, New York: Springer-Verlag, 1991.

[8] S. Gulam Razul, W. J. Fitzgerald and C. Andrieu, Bayesian model selection and parameter estimation of nuclear emission spectra using RJMCMC, Nucl. Instrum. And Methods in Physics Res. A, Vol. 497, pp. 492-510, 2003.

[9] D. Chambers, "A signal processing model for radiation transport," $L L N L$ Report, LLNL-TR-405952, 2008.

[10] J. V. Candy, E. Breitfeller, B. L. Guidry, D. Manatt, K. Sale, D. Chambers, M. A. Axelrod and A. Meyer, "Physics-Based Detection of Radioactive Contraband: A Sequential Bayesian Approach" IEEE Trans. Nuclr. Sci., Vol. 56, No. 6, pp.3694-3711, 2009.

[11] J. V. Candy, D. H. Chambers, E. F. Breitfeller, B. L. Guidry, K. E. Sale, M. A. Axelrod and A. M. Meyer, "Threat Detection of Radioactive Contraband Incorporating Compton Scattering Physics: A Model-Based Processing Approach," LLNL Report, LLNL-JRNL-422429, 2009.

[12] A. Papoulis and S. Pillai, Probability, Random Variables and Stochastic Processes, 4th ed., New York, New York: McGraw-Hill, 2002.

[13] J. Liu, Monte Carlo Strategies in Scientific Computing, New York, New York: Springer-Verlag, 2001.

[14] D. Middleton, An Introduction to Statistical Communication Theory, New York, N.Y.: McGraw-Hill, 1960.

[15] A. Wald, "Sequential tests of statistical hypothesis," Ann. Math. Stat., 16, pp. 117-186, 1945.

[16] A. Wald, Sequential Analysis, New York, N.Y.: John Wiley, 1947 (Reprint Dover Publications, 1973).

[17] M. S. Arulampalam, S. Maskell, N. Gordon and T. Clapp. tutorial on particle filters for online nonlinear/non-gaussian Bayesian tracking, IEEE Trans. Sig. Process., Vol. 50, no. 2, pp. 174-188, 2002. 